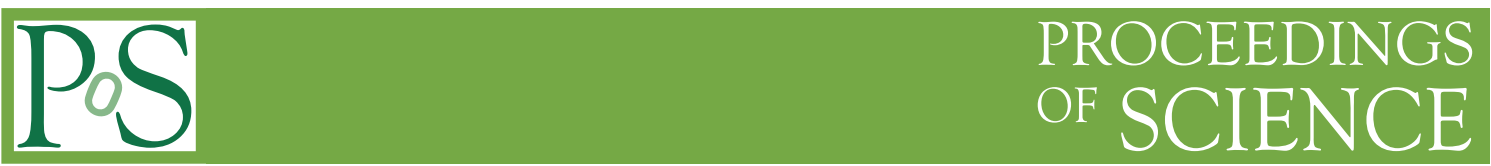

\title{
Correlated Variability in Blazars
}

\section{Robert Wagner*}

Max-Planck-Institut für Physik, Föhringer Ring 6, D-80805 München

and

Excellence Cluster Universe, Technische Universität München, Boltzmannstraße 2, D-85748

Garching

E-mail: robert.wagnerempp.mpg.de

Blazars are thought to emit highly-collimated outflows, so-called jets. By their close alignment to our line of sight, relativistic beaming effects enable us to observe these jets over the whole electromagnetic spectrum up to $\mathrm{TeV}$ energies, making them ideal laboratories for studying jet physics. In the last years multiwavelength observations of blazars provided us with detailed data sets which helped to characterize the two main components of the non-relativistic emission, peaking in the optical to X-ray and $\mathrm{GeV} / \mathrm{TeV}$ energy region, respectively. In leptonic acceleration models, they are explained by synchrotron radiation of electrons and inverse-Compton emission from the same electron population and thus, correlations of both emission regimes are expected. We review recent observational results on the presence and absence of such correlations in blazars, and discuss constraints on emission models by quantitative correlation analyses.

Workshop on Blazar Variability across the Electromagnetic Spectrum April 22-25 2008

Palaiseau, France

\footnotetext{
* Speaker.
} 


\section{Blazars}

Blazars are the most extreme class of active galactic nuclei [1]. They are thought to have jet structures well-aligned with our line of sight. Blazars emit their bulk luminosity in the $\gamma$-ray band and are characterized by high temporal variability. These short timescales are interpreted by corresponding emission regions being as small as few light days or even light minutes only, moving relativistically down the jet. Blazar spectra are dominated by non-thermal emission, and two broad, well-defined components can be identified. The low-energy peak is commonly explained by synchrotron emission from relativistic electrons in the jet, and observational evidence, e.g., characteristic emission patterns, but also substantial polarization, is given. The high-energy peak is explained in leptonic acceleration models with inverse-Compton (IC) emission from the same electrons. Particularly for blazars very often Synchrotron Self-Compton (SSC) [2] models are considered (as opposed to external-Compton models, [3]), which assume the synchrotron photons as the target field for IC process and the absence of additional strong contributions by external photon fields. This assumption seems reasonable for the BL Lac-type blazars, which often have almost featureless optical spectra, pointing to a low density of external photon fields. More generally, of course, IC radiation at $\gamma$-ray energies can originate from external photons in the jet, from external clouds, from a dusty torus, or from some other external seed photon field. Only recently have also low-peaked BL Lac objects [4-6] and Flat-Spectrum Radio Quasars (FSRQ) been detected in $E \gtrsim 75 \mathrm{GeV} \gamma$-rays [7]. Particularly in FSRQ, external photon fields must be considered. Leptonic scenarios provide a natural explanation for X-ray $-\gamma$-ray correlated variability. In hadronic acceleration models, instead, $\pi^{0}$ decays from photo-pion production, secondary electrons or synchrotron emission from protons explain the high-energy peak. In such acceleration models it is generally more difficult to accommodate X-ray $-\gamma$-ray correlations. Also, in hadronic models simultaneous $v$-emission is expected to be observed, provided sensitive enough $v$ detectors [8].

\section{TeV Blazars}

Since 1992, ground-based Imaging Air Cerenkov Telescopes have proven very successful in detecting high-peaked BL Lac objects (HBLs, see, e.g., [9] for an overview, and also [10]). The sofar best-studied blazars are Markarian (Mkn) 421, Mkn 501, PKS 2155-304, and 1ES 1959+650, particularly because they are known for some years and allowed extensive studies on various timescales. At the same time, all of the mentioned objects underwent either dramatic flares (most prominently Mkn 501 in early 1997, e.g., [11], and recently PKS 2155-304 [12]) or have rather high duty cycles (particularly Mkn 421). Not only are these objects well studied and well sampled in the $\mathrm{TeV}$ range, but also extensive monitoring and multi-wavelength campaigns have been carried out on them and are still ongoing. Note, however, that VHE outbursts do not seem to be a characteristic property of the $\mathrm{TeV}$ blazars in general, and quite some of the objects discovered since 2002 have not yet shown significant variability at all [9]. The TeV HBLs are interesting laboratories for correlation studies as they offer the possibility of observing the $\gamma$-ray emission by VHE electrons $\left(\gamma \approx 10^{7}\right)$ coupled with X-ray observations. 


\section{SSC Framework}

With generally only weak soft-photon fields in their jet environment, the high-energy peak of HBLs can be described well by one-zone or two-zone SSC models. Then, in SSC models, simultaneous observations of $\mathrm{X}$-rays and $\gamma$-rays are expected to reveal close correlations of both wavebands, as the corresponding photons are produced by electrons of similar energies. When, e.g., assuming a magnetic field in the acceleration region of $B \approx 0.1 \mathrm{G}$ and a Doppler factor $\delta \approx 10$, $1 \mathrm{keV}$ photons are emitted by electrons with $\gamma \approx 10^{6}$. The very same electrons will up-scatter photons to $E \sim \gamma m c \sim 1 \mathrm{TeV}$. Within these standard parameters, the IC up-scattering takes place in the Klein-Nishina regime. (To occur in the Thomson regime, substantially larger values of $\delta$ are required.) Within the SSC model, simultaneous variability or slight lags for low-frequencies are expected, accommodated by forward/reverse shocks [13, 14]. A lag of the $\mathrm{TeV}$ photons with respect to the X-ray synchrotron radiation can be explained by the corresponding IC seed photons (in the optical-UV regime because of Klein-Nishina suppression of higher-energy photons) becoming available only after the X-ray synchrotron photons [13]. No such lag is expected in case external seed photons are involved. Note that also light-travel time effects may lead to time lags in the SSC scenario.

A linear correlation is generally expected during flare decay, governed by cooling of the emitting electrons; the seed photons cool on longer timescale and IC photons should trace the electron cooling.

Many factors may cause washed-out correlations [15], particularly if the emission originates from small and localized acceleration regions, like from shocks [16]: One may expect (1) gradients in the highest electron energies, (2) a superposition (pile-up) of many rising and decaying flares, (3) that electrons of different energies populate different volumes (4) that the flare peak is reached earlier at higher frequencies due to a varying optical thickness.

\section{Observational Caveats}

Correlation between X-ray and $\gamma$-ray emission may provide essential information on the acceleration mechanism and the emission properties producing variability. The observational challenge, however, is to study two light curves obtained simultaneously by different instruments at largely different wavebands. Depending on the availability of instruments, the requirement of simultaneity might not be strictly achievable. Further on, ideally the sampling rate of the instruments involved should be similar. Particularly the high-energy emission $\left(E_{\gamma} \gtrsim\right.$ few $\left.100 \mathrm{MeV}\right)$ of blazars, is characterized by very short variability timescales reaching down to few minutes in both the X-ray [17] and $\gamma$-ray [18, 12] regimes. Therefore, not only an even, but certainly also a dense sampling is mandatory.

Systematic studies - SSC model tests. Finally, for any interpretation it is crucial to obtain additional spectral information to understand the spectral evolution: Systematic analytical studies [19] of the most simple case of pure SSC scenarios have been carried out assuming a spherical homogeneous source that may undergo expansion and compression, changes of the magnetic field, of the electron density, and adiabatic cooling. Particularly the cases (1) of an expanding source 
with constant particle density and magnetic field, (2) of an increasing particle density while keeping volume and magnetic field constant, (3) an increasing volume with adiabatic cooling, and (4) a decrease of particle density while the source is shrinking, the magnetic field is decreasing and adiabatic cooling is enabled. Fig. 1 shows how correlations are modified by different underlying processes in the acceleration region, but also how they depend on the position of the bandpass in both X-ray and $\gamma$-ray regimes at which the light curves are obtained.

Generally, in SSC models different processes may be considered responsible for changes in the photon flux [19]. Quadratic correlations can be produced by increasing the electron density in the source; but these are problematic to sustain during the decay phase on short timescales. This is because radiative cooling can only affect high-energy particles. Note also that strictly a quadratic correlation is only possible for inverse-Compton processes that take place in the Thomson regime, in which unacceptably large Doppler factors are implied [20]. In the Klein-Nishina regime, however, effectively the low-energy electrons producing the soft seed photons and the high-energy electrons producing the high-energy electrons are decoupled. Thus, by providing a constant seed population of low-energy photons, a linear correlation may be achieved [19]. Rather special and physically unlikely conditions are needed to construct a quadratic correlations during decay instead, e.g., adiabatic expansion of the acceleration region in combination with a constant magnetic field. Similarly, different source geometries allow for quadratic decay solutions only with these rather particular physics conditions. Note that while light-crossing time effects (LCTE) in the source may weaken intrinsic correlations, substantial external LCTE may, e.g., modify a quadratic correlation to be observed as a stronger-than-quadratic one.

\section{Observed Correlations}

Significant evidence of correlated variability has been first reported for the bright blazars Mkn 421 [21] and Mkn 501 [22], over observation windows of 15 and 11 days, respectively. Correlated variability was afterwards observed during strong flares from Mkn 421, e.g., [23--25], and from Mkn 501 [26]; over extended periods of weeks to months [27-30]. These findings were confirmed on other occasions. But correlations were found to be absent in some cases, most notably in the 2002 "orphan TeV flare" in 1ES 1959+650 [31]; also in Mkn 421 orphan TeV flares and their counterparts, "childless X-ray flares" [29] were observed.

Comprehensive studies have been carried out by [32] on Mkn 501 by applying a time-dependent SSC code on a 2-month data set, albeit with a rather sparse sampling; by [19] by systematically taking into account bandpass effects and changes in different SSC parameters to account for temporal flux variations as discussed above; and by an analysis of a week-long, densely-sampled simultaneous data set of Mkn 421 in 2001 [25].

\subsection{Correlations on Timescales of Weeks to Months}

The Mkn 501 flare in April 1997. Mkn 501 underwent a dramatic flare in the first months of 1997 , becoming the brightest $\gamma$-ray source at that time. That flare was also exceptional as the synchrotron peak energy exceeded $100 \mathrm{keV}$ [22] at peak flare times, and sustained a peak energy of $\approx 20 \mathrm{keV}$ even in the following year [33]. Observations with the Whipple telescope $(E>300 \mathrm{GeV})$, with the OSSE instrument $(50-150 \mathrm{keV})$ on board of CGRO, and with the All-Sky Monitor (ASM) 

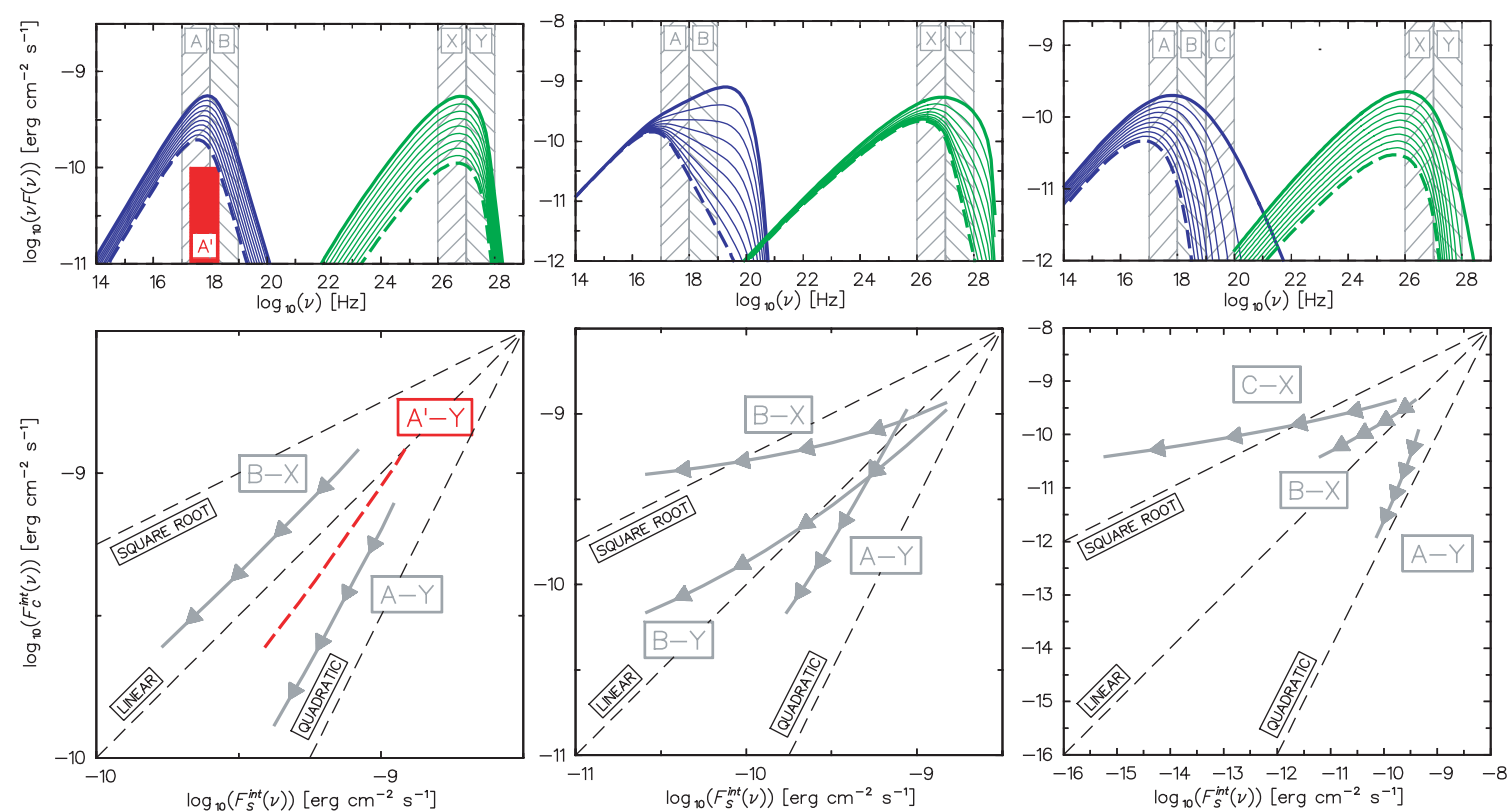

Figure 1: Left: Evolution of the SSC emission of an expanding spherical homogeneous source. The areas A, $\mathrm{B}, \mathrm{X}, \mathrm{Y}$ indicate the spectral bands used for calculating the correlation. The resulting correlations are shown in the lower panel. The bold-dashed line A'-Y shows the correlation obtained after a small shift of the Aband. Middle: Evolution of the SSC emission of the source where only the slope $n_{2}$ of the high-energy part of the electron spectrum was modified. Same spectral bands A, B, X, Y as in the left panel. Right: Impact of the radiative cooling. Taken from [19].

instrument aboard the Rossi X-Ray Timing Explorer (RXTE; $3-20 \mathrm{keV}$ ) yielded correlations of $F_{\gamma} \sim F_{\mathrm{OSSE}}^{1.7 \pm 0.5}$ and $F_{\gamma} \sim F_{\mathrm{ASM}}^{2.7 \pm 0.6}$, respectively [22]. It was necessary, however, to interpolate the Whipple and ASM data, as their sampling was less dense than that of the OSSE data. In this study also correlated optical variability was found. Observations with CAT and BeppoSAX, comprising a wider bandpass in the X-rays, $0.1-200 \mathrm{keV}$, revealed a similar correlation, $F_{\gamma} \sim F_{\mathrm{OSSE}}^{1.0 .2 .0}$ [34]. Yet another analysis [27] found a quadratic correlation between the $\gamma$-rays and X-rays measured by ASM $(3-25 \mathrm{keV})$. While a general correlation between the two energy regimes was observed, the differences in the quantitative description of the correlations is due to the positions and widths of the spectral bands involved (cf. Fig. 2).

The Mkn 421 flare in February 2000. Here measurements with the HEGRA imaging air Cherenkov telescope array $(E>1 \mathrm{TeV})$ and the Proportional Counter Array (PCA) and ASM detectors $(3-20 \mathrm{keV})$ on board of RXTE have been obtained. This time the HEGRA measurements were interpolated to match the X-ray data. A correlation $\left(F_{\gamma} \sim F_{\mathrm{X}}^{0.5 \pm 0.2}\right)$ with a rather poor $\chi^{2}$ has been found, which makes the interpretation of these results rather difficult. For this measurement, the rather sparse sampling of only one data point per day seems to be the limiting factor.

\subsection{Comprehensive campaigns}

The Mkn 421 campaign in March 2001. In 2001 March 19-25, precise observations of Mkn 421, lasting for one week, were carried out [25]. The good observation conditions in spring and the 

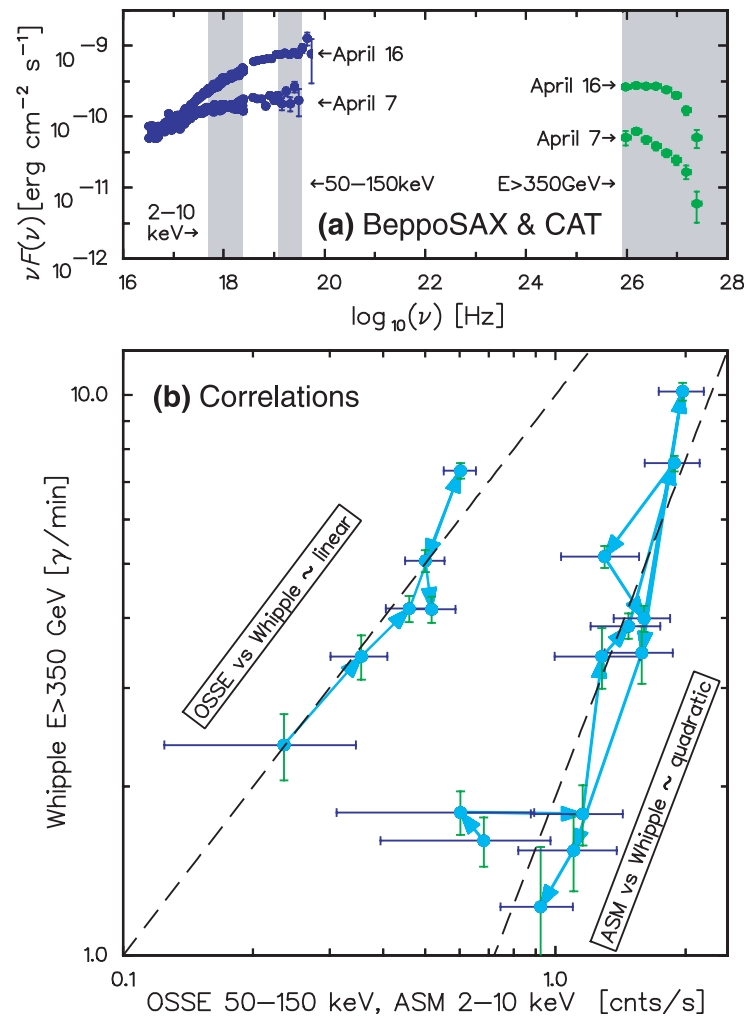

Figure 2: The activity of Mkn 501 observed in 1997 April. (a) Spectra obtained by BeppoSAX [35] and CAT [34]). The grey areas mark the bandpasses of the OSSE, ASM, and CAT instruments, respectively. (b) Two correlations between the X-ray and the TeV $\gamma$-ray fluxes. For clarity, the OSSE data in (b) were multiplied by 0.5 . The dashed lines show templates for a linear and a quadratic correlation. Taken from [19].
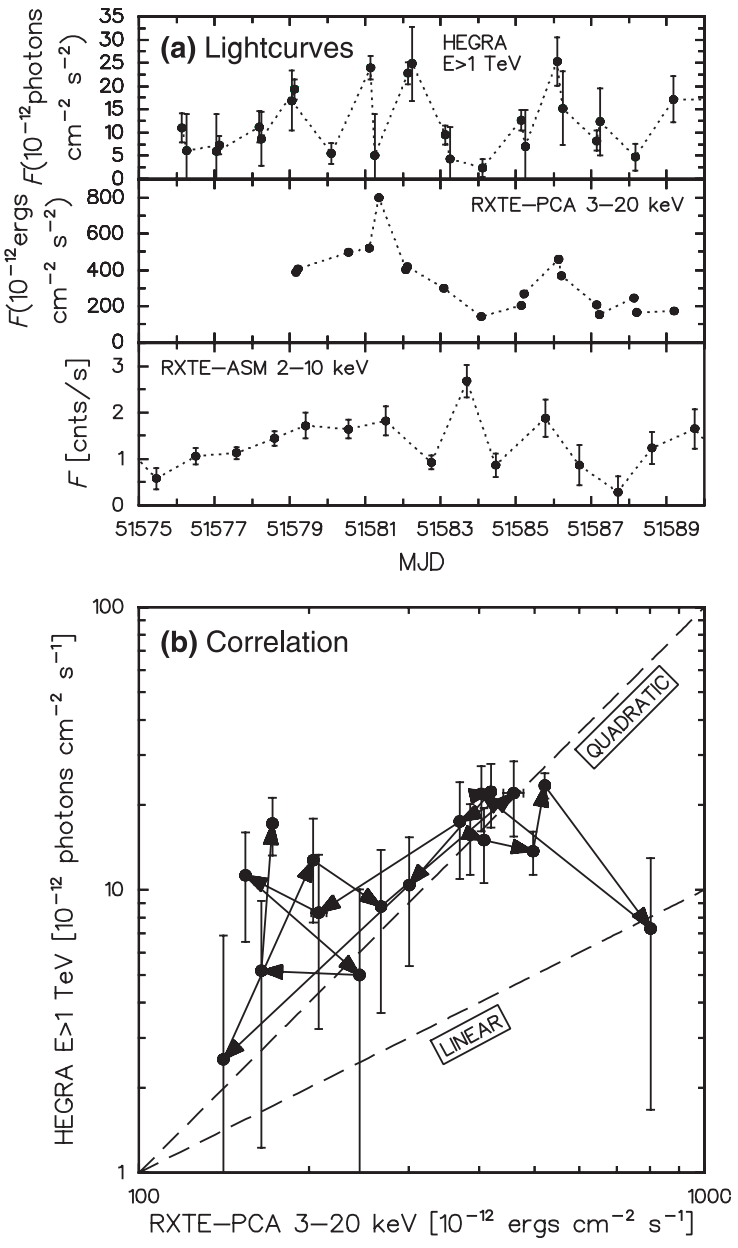

Figure 3: The activity of Mkn 421 observed in 2000 February. (a) $\gamma$-ray and X-ray light curves obtained by HEGRA, RXTE-PCA, and RXTE-ASM [36]. (b) Correlation between the X-ray and the $\mathrm{TeV} \gamma$-ray fluxes. The dashed lines show templates for the linear and the quadratic correlation. Taken from [19].

combination of data from the Whipple and HEGRA detectors, some hours apart in longitude (cf., e.g., [37]), allowed for a dense sampling in $\mathrm{TeV} \gamma$ rays. The PCA instrument aboard RXTE observed for a total of 62 hours in the $3-20 \mathrm{keV}$ range during that week. Generally, clear correlations were observed. Fig. 4 shows two of the observation nights. During the night of 2001 March 18/19 (Fig. 4a) flare could be for the first time followed from its onset to its end with sensitive instruments in both energy bands. For correlation studies a total of 105 data pairs is available. Clear correlations between the $\mathrm{TeV}$ band and various band passes ranging from 2 to $60 \mathrm{keV}$ in the $\mathrm{X}$-ray regime were found, which generally exhibit linear correlations. Also the night-by-night averages ( 7 data points) follow a linear correlation. The regions, however, which are covered in the $F_{\mathrm{TeV}}-F_{\mathrm{X}}$ plane shift from night to night, and within these night-by-night observations also quadratic correlations are found (Fig. 5). The fact that the correlations on day-scale and sub-day scale differ might be related 

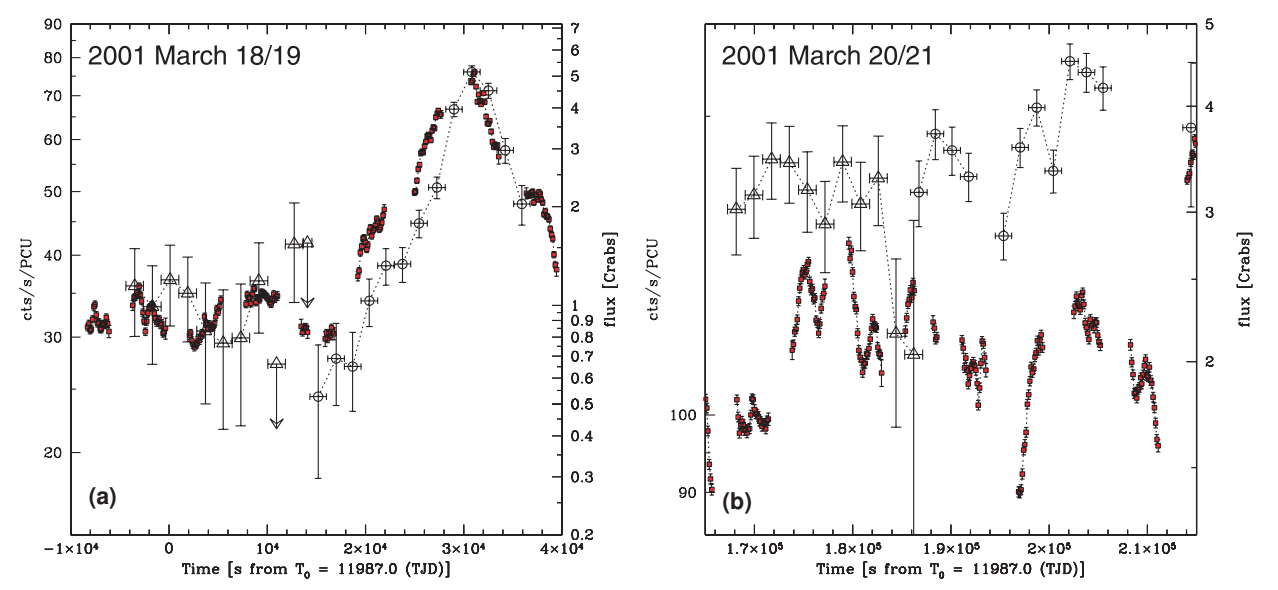

Figure 4: Two nights from the 2001 March campaign on Mkn 421. Open symbols: TeV data (triangles: HEGRA; circles: Whipple). Filled symbols: PCA data. Strong, highly-correlated variability in both energy bands is found. Taken from [25].

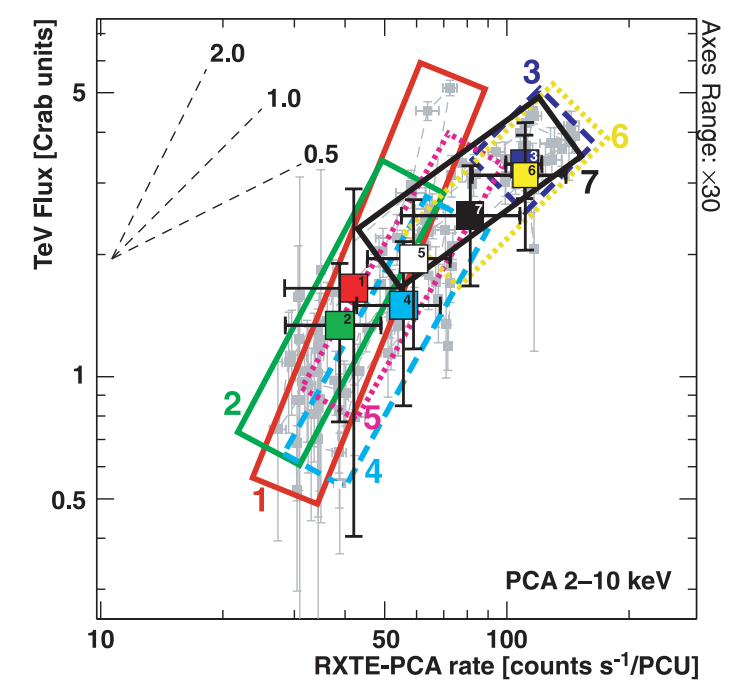

Figure 5: One-day averaged $\gamma$-ray vs. X-ray flux. The overall correlation is approximately linear. Numbers refer to the campaign-night sequence. The boxes approximately represent the regions of the diagram occupied by the data of each respective night. The combination of steeper intra-night and flatter longer-term (due to the shift of the "barycenters") correlations is seen. Taken from [25].

to more complicated variability patterns, as expected, e.g., from a pile-up of multiple flares and thus from superimposed rise and decay phases. An alternative explanation for the complex picture may also be given by two luminosity-related regimes between which the emission switches depending on the emission level.

A temporal analysis generally revealed no time lags, while a high level of correlation, $r=0.8$, was found (Fig 6a). Particularly for the March 18/19 flare it is likely that in fact an undiluted (no unresolved pile-up of many flares) has been detected. When analyzing the flare night only, a lag 

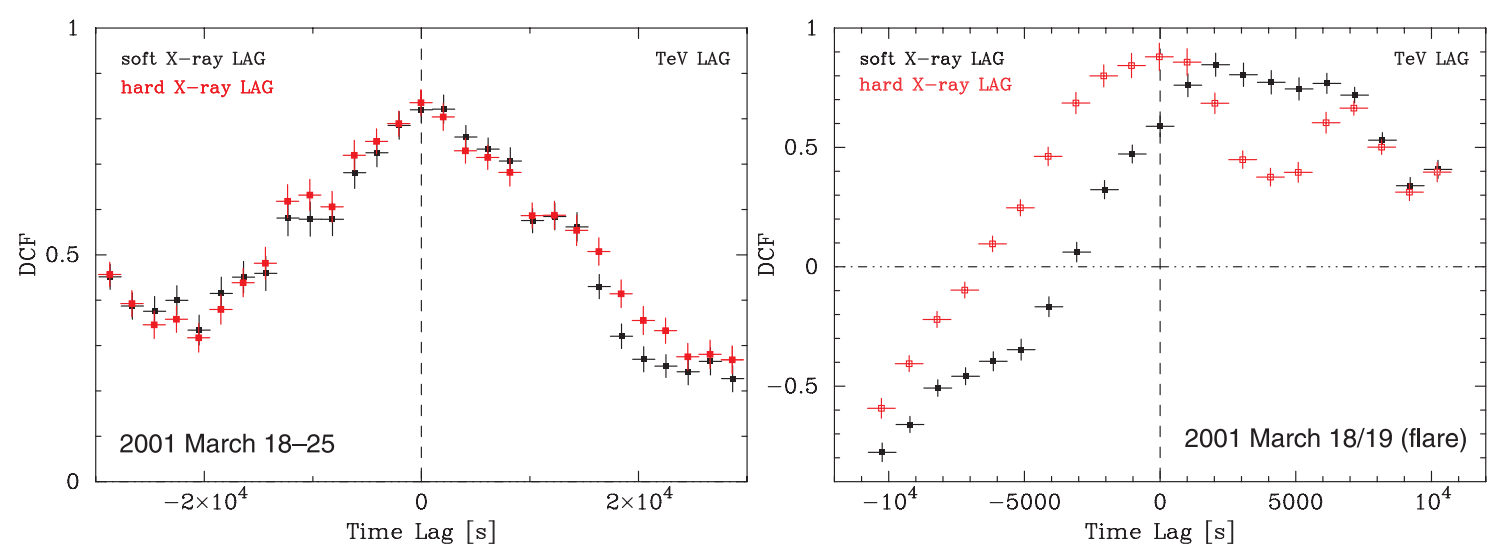

Figure 6: Cross-correlation between the $\mathrm{X}$-ray and the $\mathrm{TeV}$ light curves. (a) $2-4 \mathrm{keV}$ vs. $\mathrm{TeV} \gamma$-rays (filled, black symbols) and 9-15 keV vs. $\mathrm{TeV}$ (open, red symbols) for the whole campaign. (b) 2-4 keV vs. $\mathrm{TeV}$ (filled, black symbols) and 9-15 keV vs. TeV $\gamma$-rays (open, red symbols) for the flare night of $2001 \mathrm{March}$ 18-19. Taken from [25].

of $2 \mathrm{ks}$ for $\gamma$-rays w.r.t. to soft X-rays is found (Fig. 60 ), while the time lag w.r.t. hard X-rays is compatible with zero.

A significantly steeper correlation is found for the flare night of March 18/19, during which the $\mathrm{TeV}-\mathrm{X}$ correlation is almost quadratic during the rising and decay phase of the flare. Note that particularly during the decay phase a quadratic correlation is difficult to achieve in SSC scenarios, if the flare decay is governed by cooling of the emitting electrons. The overall Whipple-PCA correlation is $F_{\gamma} \sim F_{\mathrm{X}}^{1.3 \pm 1.0}$. The long-term correlation differs from the flare correlation. Another 1-week study [38] of Mkn 421 in 1998 yielded $F_{\gamma} \sim F_{\mathrm{X}}^{1.7 \pm 0.3}$, where the X-ray flux was measured at the synchrotron peak position.

\subsection{The Orphan Flare Case}

In 2002, strong flares from 1ES 1959+650 were observed [31] (Fig.77). Optical, X-rays (RXTEPCA), and $\gamma$-rays (Whipple) in general showed a good correlations. Particularly also a tight correlation between the 3-25 keV spectral index and the $10 \mathrm{keV}$ flux was found (Fig. 8), pointing to a synchrotron peak moving towards higher peak energies with increasing flux. However, the data set also shows clear evidence for an "orphan flare", i.e. a TeV flare without any obvious counterpart in the X-ray band. Such flares can be explained in hadronic acceleration models, and it was noted that there was a preceding X-ray flare about 15 days before the $\mathrm{TeV}$ flare, with the $\mathrm{TeV}$ flare possibly being connected to that earlier event. A couple of emission models have been discussed in the literature [31, 39, 40], one of them invoking broad-line region clouds as mirrors for the primary flare, and subsequent secondary flare from $\mathrm{p} \gamma$-interactions.

\subsection{Long-term Studies: Timescales of Years}

Mkn 421 in the 2003/4 Observing Season. The Whipple telescope observed Mkn 421 regularly during dark time in the 2003/4 season, for about 28 minutes per observation, with more runs on occasion [28]. In an analysis of 306 such runs a rough correlation was found with RXTE-PCA Xray data. As both the Whipple and PCA energy bands are rather broad, this weak correlation likely 


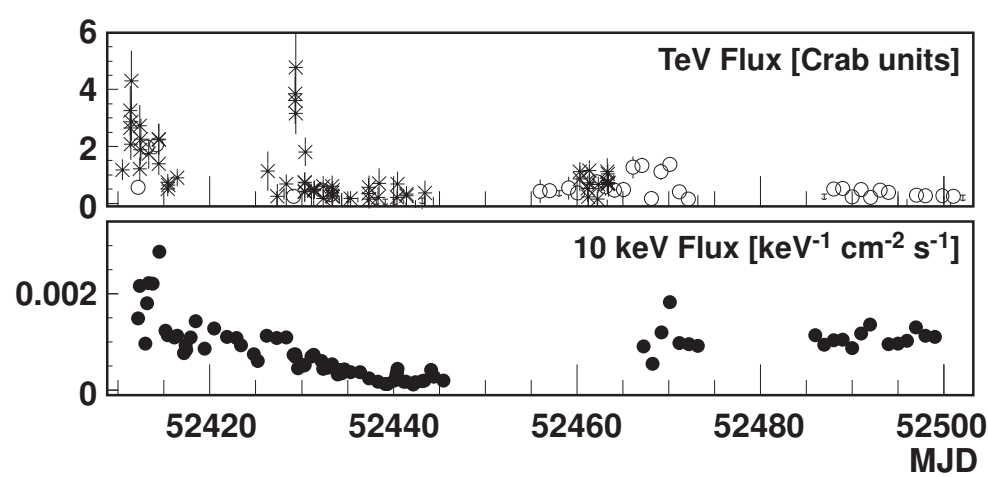

Figure 7: Results from the 1ES 1959+650 multiwavelength campaign (2002 May 16 - August 14), taken from [31]. Top: Whipple (stars) and HEGRA (circles) integral TeV $\gamma$-ray fluxes; (bottom) RXTE X-ray flux at $10 \mathrm{keV}$.

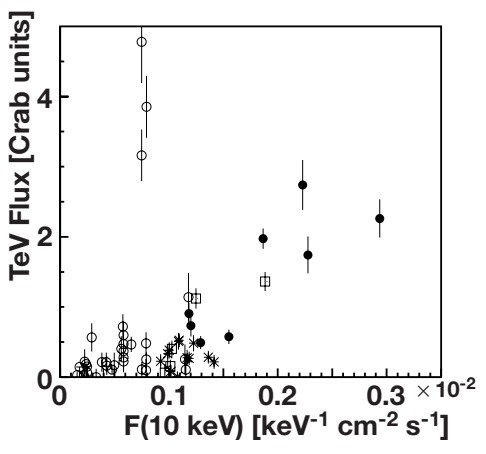

Figure 8: Correlation between the $\mathrm{X}$-ray flux and the 3-25 keV photon index. The different symbols represent different epochs of the campaign. Taken from [31].
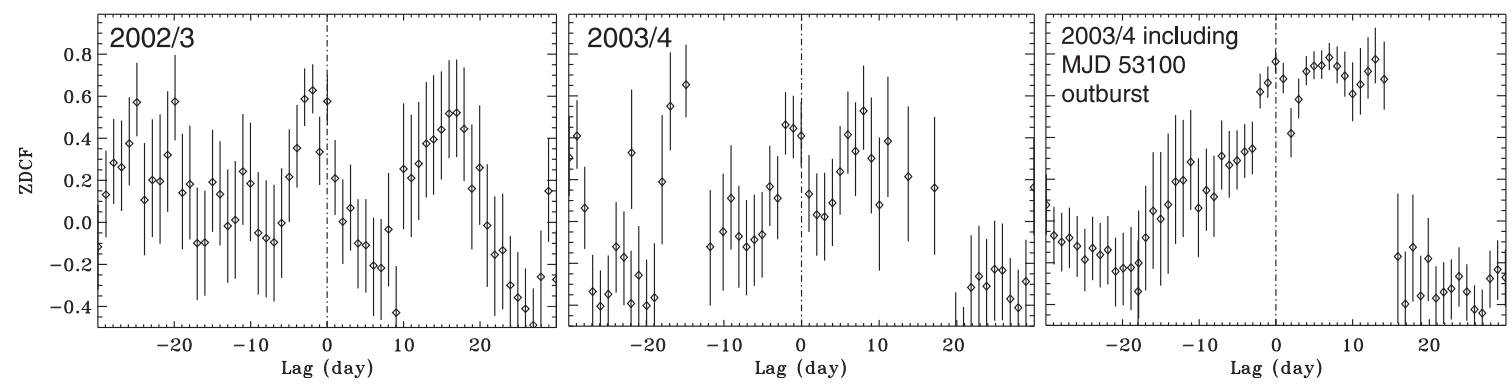

Figure 9: Z-transformed discrete correlation function between the X-ray and TeV light curves for Mkn 421 in the 2002/3 season (left), in the 2003/4 season before the giant outburst on MJD 53100 (middle), and in the entire 2003/4 season. Taken from [28].

is not an observational artifact. Despite an acceptable dynamical range 30 in both energy bands and the broad spectral coverage, the correlation more loose than expected. The discrete correlation function shows a marginal lead of the X-ray w.r.t. the $\gamma$-ray light curve of $1.4 \pm 0.8$ days (Fig. 9) both in the 2002/3 observing season and in the 2003/4 season before an outburst on MJD 53100 . When including this flare, however, the picture is modified by the different $\mathrm{X}$-ray and $\gamma$-ray rise times of the flare. The flare itself can be categorized as "orphan $\mathrm{TeV}$ flare" without simultaneous counterpart, where a candidate X-ray counterpart may have peaked 1.5 days earlier (Fig. 10).

Mkn 501 during 1997 - 1999. Mkn 501 was studied over a period of 3 years from 1997 to 1999 [41, 42]. Like for Mkn 421, a sampling every 3 to 4 days over longer observing periods was performed. For this study, contemporaneous data not more than 8 hours apart, were used. While a general positive trend was observed, a rather large scatter (Fig. 11a) points to the presence of uncorrelated activity; note also that one data point is $11 \sigma$ off the correlation (Fig. 11b). In this study also a general correlation (Fig. 11c) over various observation periods was established. When considering individual subperiods, a heterogeneous behavior is found (Fig. 12): While in 1997 May a 


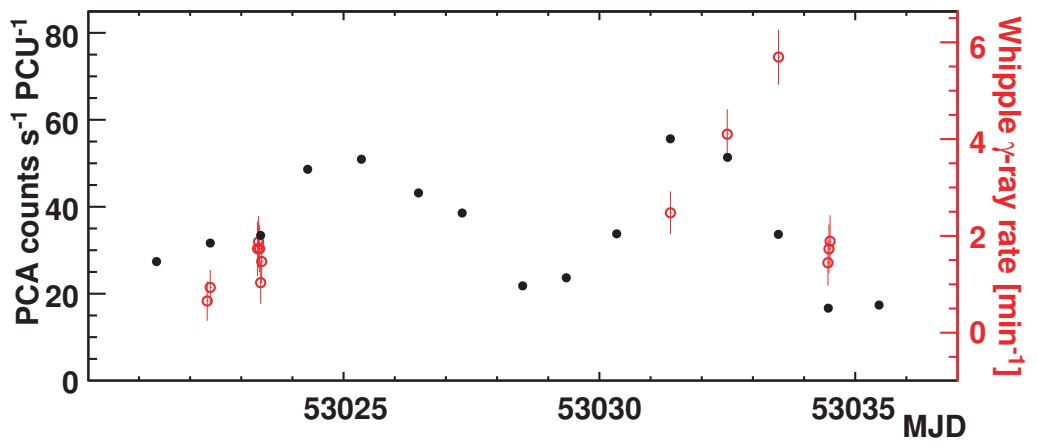

Figure 10: A TeV (red, open circles) flare in Mkn 421 without simultaneous X-ray counterpart. The (black, filled dots) X-ray PCA light curve underwent significant variability and peaked $\approx 1.5$ days before the TeV flare. Taken from [28].
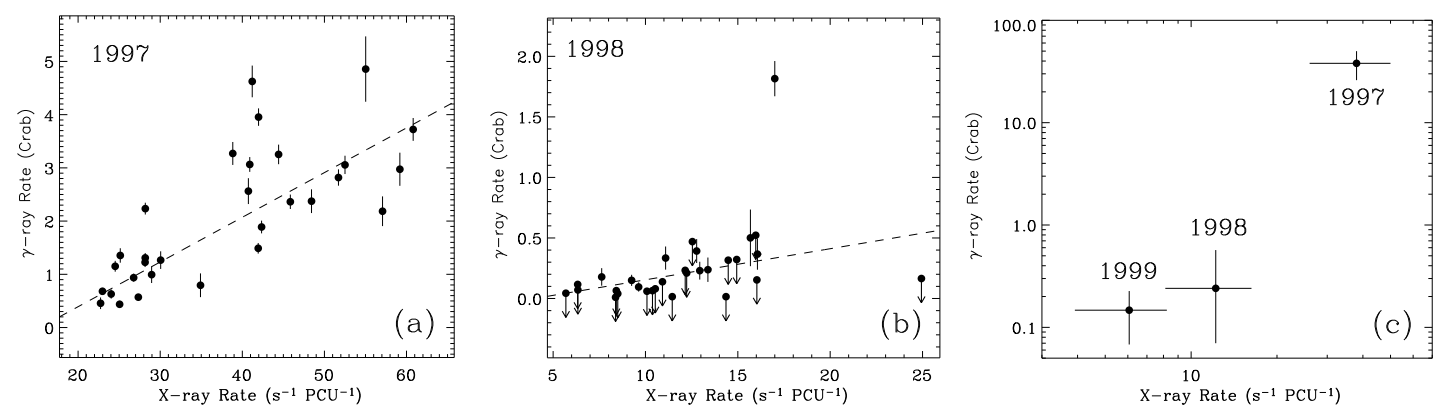

Figure 11: Mkn $501 \gamma$-ray vs. the 2-20 keV X-ray count rate during (a) 1997 and (b) 1998, respectively. (c) Mean values of the $\gamma$-ray fluxes (calculated using all data sets) vs. X-ray count rates; the horizontal and vertical error bars represent the dispersion around the X-ray and $\gamma$-ray mean values. Taken from [29].

linear correlation is seen, $F_{\gamma} \sim F_{\mathrm{X}}^{0.99 \pm 0.01}$, in 1998 June the correlation is quadratic, $F_{\gamma} \sim F_{\mathrm{X}}^{2.07 \pm 0.36}$. Note that for 1999 May not enough data are available for claiming any correlation. In fact, a timedependent modeling of Mkn 501 spectra [32] requires a two-zone SSC model, implying that more complex correlations may be expected. Note also that in the 1999 observations a "childless" X-ray flare (without $\mathrm{TeV}$ counterpart, that is), was found [209].

\subsection{Recent Campaigns}

The unprecedented sensitivity of third-generation ground-based instruments [10] allows for detailed studies of correlations by a dense sampling both in the X-ray and VHE bandpass. Particularly during flares, now light curves with a time resolution of minutes are achieved. A multiwavelength campaign on the Southern source PKS 2155-304 during 2003 October 19 to November 26 with strictly overlapping data sets from the High Energy Stereoscopic System (H.E.S.S.) and RXTE-PCA revealed no correlation [42], although intra-night variability in the VHE band was found as well as an $\mathrm{X}$-ray transient lasting $\approx 1500 \mathrm{~s}$. A reason for the non-observation of a correlation might be the rather small dynamical range of the observations. In another multi-wavelength campaign in 2004 [43], a correlation between the $>200 \mathrm{GeV}$ and the $2-10 \mathrm{keV}$ band was found with a correlation coefficient of $r=0.71 \pm 0.05$. On 2006 July 28, PKS 2155-304 underwent a 

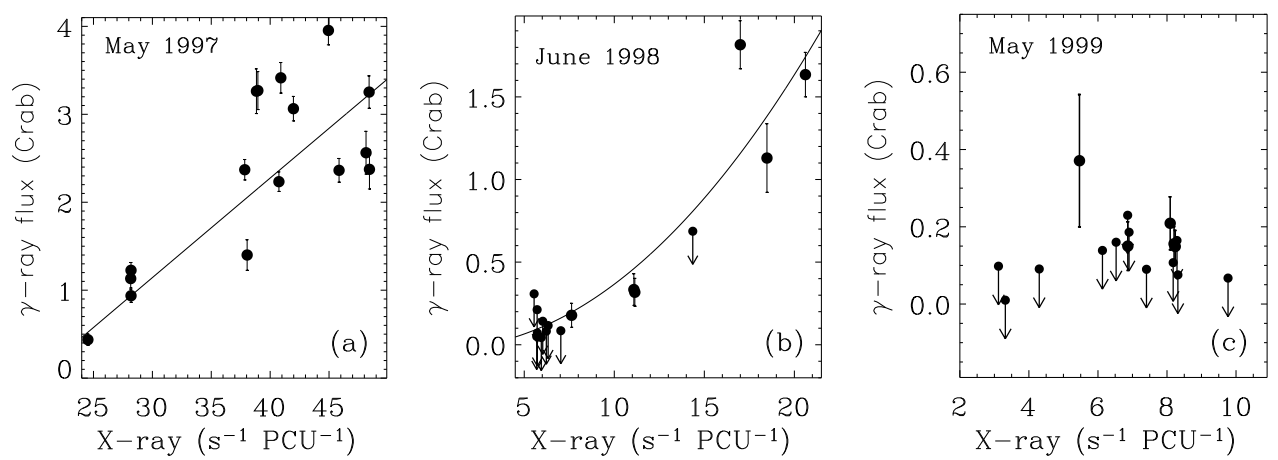

Figure 12: Mkn $501 \mathrm{TeV}$ flux vs. $\mathrm{X}$-ray count rate for three representative flares, showing (a) a linear correlation $F_{\gamma} \propto F_{\mathrm{X}}^{0.99 \pm 0.01}$, (b) a nonlinear correlation $F_{\gamma} \propto F_{\mathrm{X}}^{2.07 \pm 0.36}$, and (c) no correlation at all due to a rather low source flux and predominantly upper flux limits only. Taken from [29].

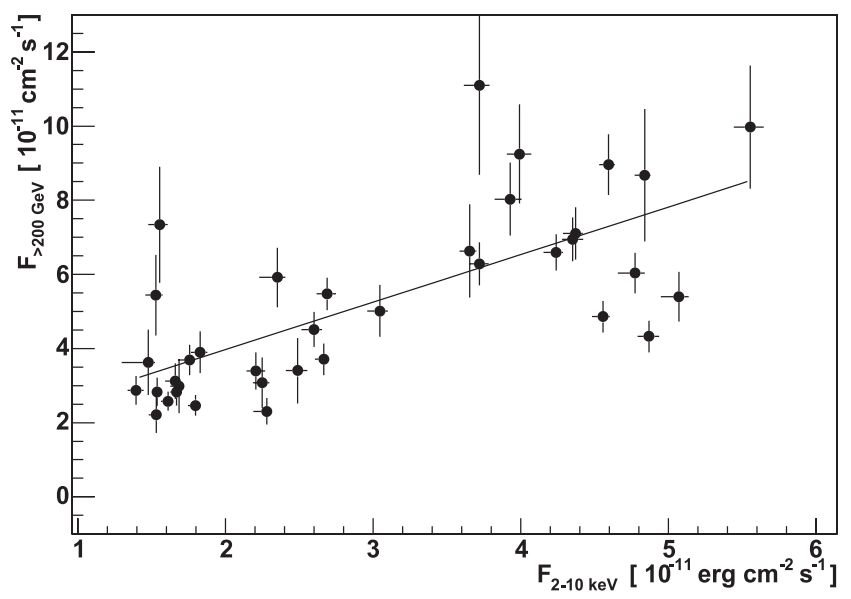

Figure 13: VHE vs. 2-10 keV X-ray flux for PKS 2155-304 in the 2004 multi-wavelength campaign during a $\gamma$-ray high state. The correlation factor is $r=0.71 \pm 0.05$. Taken from [4.3].

dramatic flare, reaching a peak level of about 15 times the Crab nebula flux [12]. The overall flare complex that was recorded lasted for about 60 minutes, with distinct sub-flares with doubling times on the minute-scale. Two nights after the reported flare, another outburst could be recorded, higher in peak intensity than the first flare, this time with simultaneous coverage in the X-ray energy band by Chandra (the "Chandra night"). Among a wealth of interesting observational facts [4] ], a correlation of the X-ray and VHE data was without any time lag. The optical flux followed the VHE flux with a longer time constant. The variabilities relate as $\Delta_{\mathrm{VHE}}: \Delta_{\mathrm{X}}: \Delta_{\mathrm{vis}} \approx 14: 2: 0.15$. Very surprisingly, an almost cubic correlation $F_{\gamma} \sim F_{\mathrm{X}}^{\alpha}, \alpha \approx 3$, was found during the flare decay phase [10], which practically rules out a simple one-zone SSC model for that observations.

In summer 2005, the Major Atmospheric Imaging Air Cerenkov (MAGIC) telescope set out for an unbiased six-week fixed-window observation of Mkn 501 [18]. The measured flux of Mkn 501 varied between $\approx 30 \%$ and $\approx 4$ Crab units. As Mkn 501 was found on a moderate flux level, a day-by-day correlation with the RXTE-ASM data revealed a marginal correlation (dominated by the large errors of the ASM-measured X-ray flux). 
Also recent measurements of Mkn 421 confirm the existence of a correlation: In 13 nights from November 2004 to April 2005, MAGIC and ASM observed a correlation [45], as did, with the mores sensitive X-ray instrument PCA, VERITAS during 14 hours from 2008 January 8 to February 13 [46]. This data set provides 11 strictly overlapping X-ray/TeV data points, or, when relaxing the simultaneity requirement by $\approx 5$ hours, 16 data points; a correlation coefficient of $r=0.76$ is reached.

While for Mkn 421 and during high-flux episodes also for Mkn 501, ASM X-ray data may be used for correlation studies, the ASM instrument generally is not sensitive enough to allow such studies for other sources (as demonstrated by [47]). For the historically third-detected TeVdetected blazar, 1ES 2344+514, only few observational data exist, but a 3-month detection in a rather low state was achieved by MAGIC in 2005 [47]. From October 2007 to January 2008 simultaneous VERITAS and RXTE-PCA observations were conducted and resulted in a detection of a simultaneous $X$-ray $/ \gamma$-ray flare in that source; the correlation coefficient for $X$-ray $/ \gamma$-ray flux was found to be $r=0.62$ [49]. Interestingly, in the latter observations an emission level quite compatible with the MAGIC measurements was found.

The first non-blazar object detected in VHE $\gamma$-rays is the close $(16 \mathrm{Mpc})$ Fanaroff-Riley I-type radio galaxy M 87. This object is believed to be a misaligned blazar [48], showing observational properties (e.g., short-timescale variability) similar to blazars. For M 87 the location of the VHE emission is still uncertain: Both the region very close to the central engine, and the brightest knot in the resolved jet, called HST-1, are considered. Note also that specific emission models for the misaligned-blazar case have been developed (e.g., [50]. See [51] for an overview). A $\gamma$-ray variability timescale as short as 1 day [52, 51] narrows down the size of the emission region to be on the order of the light-crossing time of the central blach hole, implying a production region in the immediate vicinity of the M 87 core. During observations performed with a similar sensitivity, no significant flux variations were found [53]. For M 87, an X-ray - VHE $\gamma$-ray correlation is expected in most emission models, but was not unambiguously found so far. Whereas [52] claim a hint of a correlation between the soft (0.3-10 keV) X-rays at HST-1 and the VHE $\gamma$-rays, [53] instead find a year-by-year correlation between the $(2-10 \mathrm{keV}) \mathrm{X}$-ray flux of the M 87 core and the VHE $\gamma$-ray emission, but do not observe a correlation between the two energy bands on shorter timescales. Interesting new data from a H.E.S.S.-MAGIC-VERITAS campaign in early 2008 may strengthen the core-origin hypothesis of the emission, as a high-level VHE flux and flares seen during this campaign [51, 54] were accompanied by a high-level X-ray flux from the M 87 core, while HST-1 was at its historical low-level.

\section{Optical correlations, optical triggers, radio correlations}

No obvious correlations between the optical and the TeV regime were found in 1ES 1959+650 [31], Mkn 501 [55, 18], PKS 2155-304 [42], and Mkn 421 [56]. It could, however, be shown that the fractional root mean square variability amplitude obeys a power-law for Mkn 421 [56] and can thus be used as a helpful proxy. The increase in fractional variability is also seen in X-rays [57] and VHE $\gamma$-rays [18].

In recent years, however, quite some new $\mathrm{TeV} \gamma$-ray emitters have been detected following triggers indicating a high state of these sources in the optical regime (Mkn 180 [58], 1ES 1011+496 
[59], S5 0716+714 [6]; see [60] for an overview). Additionally, the low-peaked blazar BL Lacertæ [4] and the FSRQ 3C 279 [7] were successfully detected during comparatively high states in the optical. It remains to be seen whether for some of the recently-detected blazars, particularly for low-peaked objects as 3C 279, BL Lacertæ, or W Comæ [5] correlations with the optical regime can be established.

A connection of $\mathrm{TeV}$ activity and the blazar core variability measured with Very-Large-Baseline Interferometry (VLBI) has recently been established [61]. Contemporaneous observations of Mkn 421 with the CAT instrument in the TeV energy range and with VLBI in March-April 1998 resulted in no temporal changes of the VLBI jet maps, but in strong evidence for total and polarized flux variability of the VLBI core of Mkn 421 (on timescales of weeks). The correlation is not yet established on a firm and quantitative level, but the $22 \mathrm{GHz}$ VLBI core seems to be the self-absorbed counterpart of the SSC emission at higher energies. Thus, VLBI can help to map the SSC zone. Lower radio frequencies $(15,8$, and $5 \mathrm{GHz})$ are found to be unrelated to the $\mathrm{SSC}$ phenomenon, and originate likely outside the $\gamma$-ray emission zone.

\section{Summary}

The up to now best-studied objects concerning correlations of the two bumps in the spectral energy distribution of blazars all belong to the class of high-peaked BL Lac objects. These bright $\mathrm{TeV}$ blazars have been studied in great detail for about the last ten years on various timescales and during various emission-level episodes. Correlated variability up to now has been observed on timescales ranging from hours to months, up to years and seems to be an observationally established fact for the well-studied TeV blazars. Most of the results, however, still suffer from various possible experimental caveats, including the need for an as-simultaneous-as-possible time coverage and a good sensitivity of the instruments included in the observational campaigns. Last but not least, one needs some luck to be able to follow a blazar during an outburst and be able to infer the physics of the flare production and decay.

There have been, however, few campaigns, as e.g. the 2001 campaign on Mkn 421, which provided the possibility to study correlations during the rise and decay phase of individual flares; particularly also the observation of the 2006 flares of PKS 2155-304 seem to offer almost a dissecting knife for studying the quantitative imprints of the physics processes that produce flares on the correlations.

At the same time, interesting phenomena like "orphan flares" without X-ray counterparts and "childless X-ray flares" need to be explained, and more generally, a systematic understanding of lags between the two photon populations is still elusive.

While correlations of the emission on the high-energy tails of the two photon populations have been studied in great detail, lately also potential correlations of VHE $\gamma$ rays with the optical or radio emission have been investigated. Optical triggers seem to be a successful proxy to finding new $\mathrm{TeV}$ emitters, while first studies of radio- $\mathrm{TeV}$ correlations seem to help locating the region in which the SSC phenomenon takes place, profiting from the high spatial resolution that VLBI can offer. 


\section{Acknowledgments}

I am thankful for helpful comments from Fabrizio Tavecchio.

\section{References}

[1] M. J. Rees 1984, ARA\&A, 22, 471; C. M. Urry \& P. Padovani 1995, PASP, 107, 803.

[2] L. Maraschi et al. 1992, ApJ, 397, L5.

[3] C. D. Dermer et al. 1992, A\&A, 256, L27.

[4] J. Albert et al. (MAGIC Collab.) 2006, ApJ, 666, L17.

[5] V. Acciari et al. (VERTIAS Collab.) 2008, ApJ Letters in press, preprint: arXiv:0808.0889 [astro-ph]

[6] M. Teshima (MAGIC Collab.) 2008, ATel, 1500, 1.

[7] J. Albert et al. (MAGIC Collab.) 2008, Science, 320, 1752.

[8] F. Halzen \& D. Hooper 2005, APh, 23, 537.

[9] R. M. Wagner 2008, MNRAS, 385, 119.

[10] W. Benbow 2008, in these proceedings, POS (BLAZARS2008) 012.

[11] F. Aharonian et al. (HEGRA Collab.) 1999, A\&A, 342, 69.

[12] F. Aharonian et al. (H.E.S.S. Collab.) 2007, ApJ, 664, L71.

[13] M. Chiaberge \& G. Ghisellini 1999, MNRAS, 306, 551.

[14] A. Sokolov, A. P. Marscher, \& I. M. McHardy 2004, ApJ, 613, 725.

[15] A. P. Marscher 2006, ChJAS, 6, 262.

[16] A. P. Marscher \& W. K. Gear 1985, ApJ, 298, 114; M. Spada, G. Ghisellini, D. Lazzati, \& A. Celotti 2001, MNRAS, 325, 1559.

[17] M. Catanese \& R. M. Sambruna 2000, ApJ, 534, L39; Y. Xue \& W. Cui 2005, ApJ, 622, 160.

[18] J. Albert et al. (MAGIC Collab.) 2007, ApJ, 669, 862.

[19] K. Katarzyński, G. Ghisellini, F. Tavecchio, L. Maraschi, G. Fossati, \& A. Mastichiadis 2005, A\&A, $433,479$.

[20] M. C. Begelman, M. J. Rees, \& M. Sikora 1994, ApJ, 429, L57.

[21] J. Buckley et al. (Whipple Collab.) 1996, ApJ, 472, L9.

[22] M. Catanese et al. (Whipple Collab.) 1997, ApJ, 487, L143.

[23] L. Maraschi et al. 1999, ApJ, 526, L81.

[24] G. Fossati et al. 2004, NewAR, 48, 419.

[25] G. Fossati et al. 2008, ApJ, 677, 906.

[26] R. M. Sambruna \& F. Aharonian et al. (HEGRA Collab.) 2000, ApJ, 538, 127.

[27] H. Krawczynski, P. S. Coppi, \& F. A. Aharonian 2000, A\&A, 353, 97.

[28] M. Błażejowski et al. (Whipple Collab.) 2005, ApJ, 630, 130. 
[29] M. Gliozzi, R. M. Sambruna, I. Jung, H. Krawczynski, D. Horan, \& F. Tavecchio 2006, ApJ, 646, 61.

[30] T. Schweizer, R. M. Wagner, \& E. Lorenz, ApJ subm., preprint (MPP-2008-48).

[31] H. Krawczynski et al. 2004, ApJ, 601, 151.

[32] H. Krawczynski, P. S. Coppi, \& F. A. Aharonian 2002, MNRAS, 336, 721.

[33] E. Pian 2002, PASA, 19, 49.

[34] A. Djannati-Ataï et al. (CAT Collab.) 1999, ApJ, 350, 17.

[35] E. Pian, G. Vacanti, \& G. Tagliaferri, et al. 1998, ApJ, 492, L17.

[36] H. Krawczynski et al. (HEGRA Collab.) 2001, ApJ, 559, 187.

[37] D. Mazin, F. Goebel, D. Horns, R. M. Wagner, \& S. Wagner 2005, in proceedings of 29th Int. Cosmic Ray Conf., Pune (India), 4, 331.

[38] C. Tanihata, J. Kataoka, T. Takahashi, \& G. M. Madejski 2004, ApJ, 601, 759.

[39] M. Böttcher 2005, ApJ, 621, 176.

[40] A. Reimer, M. Böttcher, \& S. Postnikov 2005, ApJ, 630, 186.

[41] J. Quinn et al. (Whipple Collab.) 1999, ApJ, 518, 693; F. Aharonian et al. (HEGRA Collab.) 2001, ApJ, 546, 898.

[42] F. Aharonian et al. (H.E.S.S. Collab.) 2005, A\&A, 442, 895.

[43] B. Giebels 2007, J. Phys. Conf. Ser., 60, 127.

[44] L. Costamante 2007, in proc. of High Energy Phenomena in Relativistic Outflows workshop, Dublin.

[45] J. Albert et al. (MAGIC Collab.) 2007, ApJ, 663, 125.

[46] L. C. Reyes (VERITAS Collab.) 2008, in these proceedings, POS (BLAZARS20 08) 057.

[47] J. Albert et al. (MAGIC Collab.) 2007, ApJ, 662, 892.

[48] Z. I. Tsvetanov et al. 1998, ApJ, 493, L83.

[49] D. Horan (VERITAS Collab.) 2008, American Astronomical Society, HEAD meeting \#10, \#20.05.

[50] M. Georganopoulos, E. S. Perlman \& D. Kazanas 2005, ApJ, 634, L33; F. Tavecchio \& G. Ghisellini 2008, MNRAS, 385, L98.

[51] J. Albert et al. (MAGIC Collab.) 2008, ApJ Letters in press, preprint (arXiv:0806.0988 [astro-ph]).

[52] F. A. Aharonian et al. (H.E.S.S. Collab.) 2006, Science, 314, 1424.

[53] V. A. Acciari et al. (VERITAS Collab.) 2008, ApJ, 679, 397.

[54] M. Beilicke, M. Hui, D. Mazin, M. Raue, R. M. Wagner, \& S. Wagner 2008, in proceedings of 4th Heidelberg International Symposium on Gamma-Ray Astronomy.

[55] D. Petry et al. 2000, ApJ, 536, 742.

[56] B. Giebels, G. Dubus, \& B. Khélifi 2007, A\&A, 462, 29.

[57] G. Fossati et al. 2000, ApJ, 541, 153.

[58] J. Albert et al. (MAGIC Collab.) 2006, ApJ, 648, L105.

[59] J. Albert et al. (MAGIC Collab.) 2006, ApJ, 667, L21.

[60] E. J. Lindfors, D. Mazin, K. Nilsson \& L. Talkalo (MAGIC Collab.) 2008, in these proceedings, POS (BLAZARS2008) 047.

[61] P. Charlot, D. C. Gabuzda, H. Sol, B. Degrange, \& F. Piron 2006, A\&A, 457, 455. 